\title{
Rol Docente del Investigador en Matemática Educativa: un Ejemplo en un Curso de Posgrado para Profesores del Nivel Superior
}

\section{Teaching Role of the Researcher in Educational Mathematics: an Example in a Postgraduate Course for Teachers of Higher Level}

\author{
Jimena Fernández \\ Verónica Molfino ${ }^{* *}$ \\ Cristina Ochoviet ${ }^{* * *}$
}

\begin{abstract}
Resumen
Presentamos una metodología de trabajo en la línea propuesta en Ochoviet y Oktaç (2011), que buscando establecer vínculos entre la investigación y la práctica de la enseñanza, recupera el rol docente del investigador en matemática educativa. En particular, desarrollamos una actividad que tuvo como propósito acercar a profesores de matemática, estudiantes de un curso de posgrado, algunos resultados de una investigación desarrollada en Uruguay y reflexionar sobre su articulación con las prácticas de aula. La experiencia llevada a cabo y las producciones de los docentes participantes permitieron apreciar una comprensión de los aportes de la investigación en la que se centró la actividad, evidenciada a través de la reformulación de una clase previamente dada por ellos mismos acorde al marco teórico presentado.
\end{abstract}

Palabras-clave: Rol Docente del Investigador. Relación Investigación - Práctica de Aula. Formación de Profesores. Nivel Superior.

\begin{abstract}
We introduce a working methodology according to Ochoviet and Oktaç (2011), which recovers the teaching role of the researcher in educational mathematics, trying to establish links between research and teaching practices. In particular, we developed an activity which purpose was to provide mathematics teachers, taking a postgraduate course, with some results of a research study conducted in Uruguay and reflect on their articulation with classroom practices. The experiment was carried out and the productions of the teachers involved allowed us to appreciate an understanding of the contributions of the research around which the activity was centered, which was evidenced through the reformulation of a previously taught class according to the theoretical framework presented.
\end{abstract}

\footnotetext{
* Magíster en Enseñanza de las Ciencias Exactas y Naturales con orientación en Matemática por la Universidad Nacional del Comahue (COMAHUE). Profesora de Matemática en el Consejo de Formación en Educación (CFE) y en el Consejo de Educación Secundaria (CES), Montevideo, Uruguay. Dirección postal: Asilo 3255, Unión, 11600, Montevideo, Uruguay. E-mail: surrumbu@gmail.com

*** Doctora en Matemática Educativa por el Centro de Investigación en Ciencia Aplicada y Tecnología Avanzada del Instituto Politécnico Nacional (CICATA - IPN). Docente del Departamento de Matemática del Consejo de Formación en Educación (CFE), Montevideo, Uruguay. Dirección postal: Enrique Guarnero 3973, Prado, CP 11700, Montevideo, Uruguay. E-mail: veromolfino@gmail.com

*** Doctora en Matemática Educativa por el Centro de Investigación en Ciencia Aplicada y Tecnología Avanzada del Instituto Politécnico Nacional (CICATA - IPN). Coordinadora Académica del Departamento de Matemática del Consejo de Formación en Educación (CFE), Montevideo, Uruguay. Dirección postal: Asilo 3255, Unión, 11600, Montevideo, Uruguay. E-mail: cristinaochoviet@gmail.com
} 
Keywords: Researchers Teaching Role. Relationship between Research and Teaching Practices. Teacher Training. Tertiary Level.

\section{Introducción}

Establecer lazos entre los resultados de investigación y las prácticas de aula constituye, al mismo tiempo, un objetivo de todos los integrantes de la comunidad educativa y una preocupación, ya que es reconocido como un asunto difícil de lograr. Ante ese desafío, numerosos trabajos han desarrollado metodologías de investigación en las que investigadores y docentes trabajan en forma colaborativa (CZARNOCHA; PRABHU, 2004; MOSCHKOVICH; BRENNER, 2000; RAYMOND; LEINENBACH, 2000; DESGAGNÉ et al., 2001). No obstante, en Uruguay, un importante número de docentes en ejercicio no participa en trabajos de investigación pues el desarrollo de la investigación en matemática educativa (ME) se encuentra aún en estado incipiente (OCHOVIET, 2007).

En este artículo presentamos una metodología de trabajo en la línea propuesta en Ochoviet y Oktaç (2011), que buscando establecer vínculos entre la investigación y la práctica de la enseñanza, recupera el rol docente del investigador en ME con el propósito de enseñar a profesores de matemática, estudiantes de un curso de posgrado, algunos resultados de investigación provenientes de una investigación desarrollada en el Uruguay y su articulación con las prácticas de aula.

\section{El rol docente del investigador: contribuir a la comprensión de los resultados de investigación}

Si bien es habitual sostener que la comunicación de resultados constituye la fase final de un proceso investigativo, también hay quienes consideran que la última fase la constituye la publicación de los resultados. Otros van aún más lejos, planteando que la investigación termina cuando el lector entiende el artículo: "[...] es decir, que no bastaría con publicarlo, sino que sería necesario además que la audiencia comprendiese su contenido" (CANTORAL, 2007, p. 312).

Cuando trabajamos con docentes, el objetivo de facilitar la comprensión cobra especial importancia y merece una atención particular, al menos por dos motivos. En primer lugar, el conocimiento emergente de la investigación constituye conocimiento didáctico del contenido que es uno de los conocimientos base para la enseñanza (SHULMAN, 2005) y de 
ahí que resulte tan relevante lograr su comprensión. En segundo lugar, cuando pensamos en profesores en ejercicio que no son investigadores ni tienen formación en investigación, enfrentamos una dificultad adicional que es la poca o nula familiaridad con los marcos teóricos y metodológicos de la ME, tal como lo reportan Boero y Szendrei (1998).

Ochoviet y Oktaç (2011) señalan que si nos proponemos favorecer la comprensión de los resultados de investigación, deberíamos sumar instancias en las que los investigadores además de comunicar estos resultados, se ocuparan de su enseñanza, a través de actividades especialmente diseñadas para este fin. Proponemos, entonces, que el investigador en ME asuma el rol docente en los ámbitos de desarrollo profesional de los educadores. Asumir este rol implica, entre múltiples aspectos, la responsabilidad de diseñar los ambientes de aprendizaje que permitan, a los profesores participantes, la comprensión de las problemáticas en que se ha focalizado la investigación así como el poder establecer relaciones con las prácticas de aula.

En esa misma línea, es importante precisar que el rol docente que concebimos se aleja de posturas verticales de transmisión del conocimiento por parte del investigador. La alternativa propuesta es desarrollar proyectos de trabajo colaborativo entre investigadores y docentes que nutran las prácticas de ambas partes en su desarrollo profesional específico, y el curso que describimos en este artículo es un ejemplo de ello.

\section{El aprendizaje desde una perspectiva sociocultural: elementos para el diseño de la enseñanza}

Si estamos pensando en el rol docente del investigador con el objetivo de contribuir a la comprensión de resultados de investigación, no solamente debemos pensar en los recursos que utilizaremos para el diseño de las actividades de enseñanza, sino también en la forma en que organizaremos el ambiente de aprendizaje. Se hace necesario establecer, entonces, cómo concebimos en ese ámbito, el desarrollo de la tarea docente por parte del investigador, con el objetivo mencionado.

Montecinos (2003) analiza las tendencias en los programas de desarrollo profesional y reseña cuáles son las características que determinan que estos programas sean efectivos. Una de ellas es el trabajo en equipo, dado que el conocimiento está distribuido entre los integrantes de una comunidad profesional. Según la autora, el aprendizaje en colectivo permite dar lugar a instancias de trabajo donde los participantes discutan y reflexionen: "A través de la conversación entre colegas se generan oportunidades para que los docentes articulen sus 
conocimientos previos en un proceso de diálogo reflexivo que puede llevar a cuestionar esos conocimientos" (MONTECINO, 2003, p. 114).

Adoptamos, entonces, una perspectiva teórica que pudiera dar un encuadre al trabajo en equipos de docentes y que privilegiara la conversación entre colegas como medio para lograr aprendizajes.

Pea (1993) nos brinda elementos para pensar los procesos de aprendizaje desde una perspectiva sociocultural, y sus aportes permiten extraer elementos metodológicos para la acción docente que pensamos desarrollar. Aborda el estudio de los procesos de cambio conceptual, dando más peso a los factores sociales que a los cognitivos. Señala que el aprendizaje se construye a través de conversaciones entre las personas, que involucran la creación de comunicaciones y el esfuerzo para interpretarlas. La creación y la interpretación son los procesos de acción recíproca de la conversación humana a través de los cuales es negociado el significado de la acción simbólica que involucra el habla, el uso de representaciones como fórmulas o diagramas, o los gestos. Es así que el significado emerge a partir del diálogo o las acciones simbólicas entre dos interlocutores.

Pea (1993) explica que la negociación de significados y la apropiación son los dos mecanismos fundamentales que sostienen este proceso de aprendizaje que se da a través de la conversación. La negociación de significados se da cuando, a través de la conversación, aparecen interpretaciones confusas, pedidos de aclaraciones, de rectificaciones, de elaboraciones, de reformulaciones, de parafraseos $\mathrm{u}$ otros dispositivos lingüísticos que permiten indicar problemas en la comprensión.

El otro mecanismo, la apropiación, refiere a que el conocimiento de una determinada herramienta se adquiere a través de su uso compartido con otros integrantes de la comunidad. El conocimiento de las funciones de una herramienta no se construye por mera exploración del que aprende, sino en interacción con otros que ya conocen sus usos y funciones. En esta interacción entre integrantes de una comunidad pueden surgir otras interpretaciones que transforman la herramienta y nuestro pensamiento. A través de las interpretaciones de los demás podemos darnos cuenta de que el sentido que habíamos construido sobre algo, puede llegar a significar más de lo que pensábamos, dando lugar a interpretaciones más complejas. Asimismo, aprendemos el significado de nuestras producciones cuando aceptamos las interpretaciones de otros sobre lo que hemos dicho.

Britt, Irwin y Ritchie (2001, p.31) sugieren focalizarse en las conversaciones profesionales para lograr el desarrollo profesional. Estos autores definen las conversaciones profesionales como "discusiones entre aquellos que comparten una tarea compleja o una 
profesión con el objetivo de mejorar su comprensión sobre ella y su eficacia en lo que hacen". Al inicio de su estudio, el rol de los investigadores fue el de moderar estas conversaciones entre los docentes pero luego comenzaron a darse sin la presencia de estos y constituyeron un motor para el análisis de las prácticas y el desarrollo profesional de todos los participantes. Concluyen que estas conversaciones resultaron muy útiles para que los docentes participantes modificaran sus creencias y sus prácticas.

Asumiendo esta perspectiva del aprendizaje, concebimos el espacio de enseñanza como aquel que favorece las instancias de comunicación e intercambio entre los integrantes de una comunidad que, en nuestro caso, está conformada por profesores y cursillistas de un curso. Por ello, tanto la dinámica que se propone para la gestión de las sesiones de trabajo, en este caso en un aula virtual, como el diseño de actividades de enseñanza promueven la conversación entre los integrantes de la comunidad. Esta conversación se hace posible a través del trabajo en pequeños grupos, discusiones grupales, comunicación de los distintos puntos de vista y un diseño de actividades basado en "la pregunta como elemento que posibilita y habilita el diálogo entre los participantes, dando lugar a procesos de creación e interpretación que permitirán la emergencia de significados compartidos enriqueciendo la perspectiva de todos los participantes" (OCHOVIET; OKTAÇ, 2011, p. 64). Entendemos por comprensión el proceso por el cual cada sujeto, en interacción con otros, avanza en la construcción de significados. Avanzar implica que la negociación de significados da lugar a momentos en los que los significados son compartidos por los integrantes de la comunidad discursiva. Este proceso es dinámico en tanto nuevas interacciones pueden generar nuevos significados. El investigador responsable de llevar adelante la función docente en esta pequeña comunidad tendrá también la oportunidad de abrirse a otras miradas sobre su trabajo y de generar nuevas ideas o interpretaciones, tanto para reformular su trabajo como para abrir nuevos problemas de investigación.

\section{El contexto}

El curso en el que se enmarca la actividad que analizamos forma parte de la malla curricular de un programa de posgrado en matemática (mención aplicaciones o mención enseñanza), llevado a cabo en Uruguay. En particular, se trata de la primera cohorte del posgrado, y los 40 cursillistas son, en esta oportunidad, profesores egresados de institutos de formación docente. 
Se espera que los egresados desarrollen capacidades académicas que les posibiliten desempeñarse como profesores de matemática en la formación inicial de profesores así como en carreras de grado universitarias, generar proyectos innovadores referidos a la matemática y su enseñanza e "impactar en sus respectivos colectivos docentes como referentes capaces de aportar tanto en el conocimiento matemático como en la reflexión sobre su enseñanza" (OCHOVIET; VIEITEZ, 2012, p. 5).

El curso en el que se enmarca el ejemplo que desarrollamos es Aportes de la investigación para la enseñanza de la matemática en el nivel superior, perteneciente al núcleo B (espacios en los que se apuesta a una reflexión en la enseñanza de la matemática), ofrecido en el primer semestre de esta primera edición del posgrado (2014). El mismo

[...] tiene por objetivo presentar a los cursillistas un panorama amplio de diversos resultados relativos al aprendizaje del álgebra lineal y del cálculo. La lectura y análisis de diferentes trabajos en el campo permitirá la discusión y aportará elementos para la reformulación de la enseñanza de la matemática en este nivel (OCHOVIET; VIEITEZ, 2012, p.18).

Dos de las autoras de esta investigación fuimos profesoras a cargo del curso, es decir que nos hemos posicionado en el rol docente del investigador en ME.

El curso, de cuatro meses de duración, se dictó prácticamente en su totalidad en línea, con un encuentro presencial de una jornada completa a los dos meses de comenzado. Se articuló en torno a diez actividades que fueron recorriendo cuatro unidades temáticas: (1) Evolución de la matemática educativa como disciplina científica; (2) Proceso investigativo en matemática educativa; (3) Perspectivas acerca del desarrollo de la noción de variable; (4) Aportes de la investigación en torno a los procesos de aprendizaje y de enseñanza del Cálculo.

\section{Metodología de enseñanza utilizada en el curso}

La participación de los profesores en programas de desarrollo profesional es fundamental porque los ayuda a ampliar sus conocimientos de contenido, de conocimiento didáctico del contenido y a mejorar sus prácticas de aula (BORKO, 2004; EVEN, 1999; TSAMIR, 2008). Sin embargo, es poco lo que se ha reportado respecto a qué es lo que los formadores de profesores de Matemática deberían saber (CASTRO; LI, 2014).

Nipper y Sztajn (2008, p.339) afirman que "las iniciativas en la educación de los formadores de profesores están en sus primeras etapas de diseño, y queda mucho trabajo por hacer". 
En el curso utilizamos una metodología de enseñanza acorde al modelo propuesto en Castro y Li (2014), que si bien surge a partir de investigaciones relativas al desarrollo profesional de profesores de Matemática, fue diseñado e implementado en el contexto de un programa para formadores de profesores. El modelo consta de cuatro características centrales:

[...] el aprendizaje está basado en el contenido del aprendizaje y la enseñanza, las actividades deberían crear desequilibrio para los formadores de profesores y fomentar la colaboración entre ellos, y el aprendizaje está embebido o directamente relacionado con la tarea de enseñar a profesores (CASTRO; LI, 2014, p. 130).

Los cursillistas de este curso de posgrado eran docentes en ejercicio, tanto en nivel secundario como en la formación de profesores, en consecuencia traían a las diferentes instancias de trabajo todos los conocimientos provenientes de su formación y experiencia profesional. Entendimos que una forma de ayudarlos en el proceso de reflexión que permitiera tanto la comprensión de los resultados de investigación como una reformulación de la enseñanza en función de ellos, podía consistir en el análisis y reflexión sobre las prácticas de aula utilizando como herramienta los resultados de investigación.

Así, las tareas proponían recuperar las prácticas de aula de los docentes participantes pero al mismo tiempo reformularlas en función de esos resultados. Se daba, de esta forma, una relación dialéctica entre resultados de investigación y enseñanza: los resultados de investigación les permitieron reformular la enseñanza y, a su vez, esta reformulación de la enseñanza permitía una contextualización que abonaba a la comprensión de los resultados, en tanto permitía visualizarlos, aún en la teoría, en escena.

Para el diseño de las tareas del curso nos inspiramos en lo que proponen Markovits y Smith (2008). Estos autores sugieren el uso de casos porque entienden que es una de las formas de proveer el conocimiento necesario para la enseñanza, tanto a los profesores en formación como a aquellos que ya están en ejercicio. Señalan que aunque todavía es necesaria mayor investigación, existen resultados que ponen en evidencia que el uso de casos mejora el pensamiento pedagógico de los docentes y las habilidades de razonamiento. Consideran dos grandes categorías de casos: los ejemplares y las situaciones problemáticas. Los primeros son utilizados para presentar situaciones reales de aula donde puede reflejarse en forma vívida la situación sobre la que se desea reflexionar ya sea de naturaleza matemática o didáctica. Las situaciones problemáticas son apropiadas para analizar la complejidad de la enseñanza o la problemática del desempeño de los estudiantes. En esta última categoría ejemplifican el caso de las situaciones de la clase de matemática (SCM) que se centran en problemas específicos de la enseñanza de la matemática. Las caracterizan como situaciones de clase que involucran a la matemática y utilizan situaciones reales o ficticias que permiten analizar el pensamiento 
de los estudiantes a través de situaciones detectadas en la práctica docente o a través de la investigación.

En nuestro curso utilizamos casos ejemplares cuando solicitamos a los cursillistas, por ejemplo, analizar errores de producciones de estudiantes. También empleamos situaciones problemáticas cuando solicitamos a los cursillistas que analizaran páginas de libros de texto de enseñanza y sus implicancias para el aprendizaje. Las SCM también fueron utilizadas cuando pedimos a los cursillistas que recuperaran experiencias de prácticas de aula para situarse sobre ellas y discutieran cómo podrían haber intervenido a la luz de los documentos estudiados. La actividad que seleccionamos para reportar en este artículo podría catalogarse como SCM, pero con un leve matiz: pedimos que se sitúen en una propuesta propia de aula y la reformulen a partir de lo reseñado en resultados de investigación.

La idea de vincular el estudio de los resultados de investigación con la reformulación de la enseñanza vinculada a la práctica del profesor, se fundamenta en que los programas de desarrollo profesional logran mayor impacto cuando los docentes participantes pueden apreciar que lo que aprenden les será útil para mejorar sus prácticas (GRESALFI; COBB, 2011; GUSKEY, 2002).

\section{La actividad seleccionada}

En este trabajo analizamos las producciones relativas a una de las actividades correspondientes a la tercera unidad del curso, adjunta en el anexo. La misma fue realizada por 34 cursillistas en subgrupos de entre dos y tres integrantes, esperando de esta manera promover la construcción de significados mediante la comunicación y el intercambio de los integrantes de la comunidad de docentes cursillistas.

Elegimos esta actividad para analizar y difundir, dado que uno de los documentos que los cursillistas debían leer es Fernández (2013), tesis de maestría dirigida por una de las docentes que dictaba el curso. Por otra parte, este trabajo de tesis se llevó a cabo con estudiantes del último año de profesorado de matemática de un instituto de formación docente en Uruguay. De esta manera, contribuimos al enriquecimiento del triángulo didáctico específico que describe el rol docente del investigador, propuesto en Ochoviet y Oktaç (2011), expreso en la figura 1. 


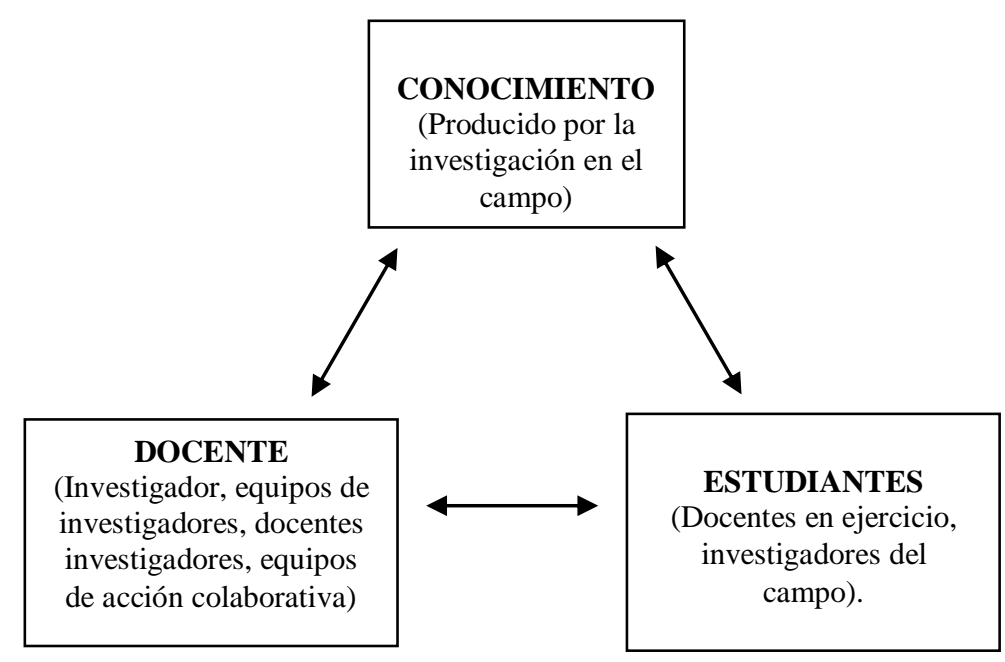

Figura 1- Triángulo didáctico relativo al rol docente del investigador

Los resultados de investigación obtenidos en Fernández (2013) son utilizados para el diseño de actividades que facilitan la construcción de significados entre el colectivo de docentes cursillistas, mediante la guía de investigadoras, en su rol de docentes del curso.

Fernández (2013, p.13) plantea como objetivo

[...] explorar y analizar el desarrollo del sentido de los símbolos que manifiestan en la resolución de diferentes situaciones donde lo algebraico está en juego, los estudiantes del último año (4to año) de la carrera de profesor de matemática de educación media en un instituto de formación docente del Uruguay.

Para ello hace uso del marco teórico desarrollado por Arcavi $(1994,2007)$ relativo precisamente a su caracterización del sentido de los símbolos.

Es por esto que para la confección de la actividad, no solo propusimos leer fragmentos de la tesis, sino además dos documentos de Arcavi $(1994,2007)$ que permitían al cursillista tener un contacto directo con marcos teóricos propios de la ME. En dichos documentos el autor describe detalladamente y ejemplifica los comportamientos que considera como los componentes más importantes que demuestran haber construido un sentido de los símbolos, aunque no descarta que pueda haber otros.

Arcavi señala que los estudiantes no desarrollarán sentido de los símbolos si no son capaces de llevar de forma flexible los significados de los símbolos que manejan hacia otras representaciones. El poder moverse a través de las diferentes representaciones enriquece la comprensión de cada una de ellas; ser capaz de trabajar con otras representaciones enriquecerá el desarrollo del sentido de los símbolos del individuo.

\section{Diseño de la actividad propuesta a los cursillistas}


La actividad (ver Anexo 1) se compone de preguntas críticas (en el sentido de WASSERMAN, 1999) que promueven conversaciones entre los miembros de cada subgrupo con el fin de favorecer la negociación de significados y la apropiación, generando aprendizajes desde la perspectiva sociocultural propuesta por Pea (1993).

Desde este punto de vista, el ítem 2 de la actividad invita a una negociación del significado del sentido de los símbolos. La finalidad es que los integrantes del subgrupo discutan sobre lo que cada uno interpreta de los documentos de Arcavi (1994, 2007), analizando los conceptos y ejemplos allí desarrollados. El máximo en la extensión se propone con un doble objetivo; el primero, orientativo, para que los cursillistas tengan una mejor idea de cuánto se espera profundicen en ese ítem. El otro objetivo apunta a la calidad de la respuesta, obligando a los cursillistas a jerarquizar lo esencial del marco teórico, discutir sobre posibles interpretaciones confusas para generar la negociación de significados al decir de Pea (1993).

Se pretendía que explicitaran que para Arcavi esta no es una noción con una definición acabada, sino que se pueden enumerar una serie de comportamientos, pero no en forma exhaustiva. Además, se esperaba que desarrollaran para qué considera Arcavi que puede ser usado el constructo (a la interna de la disciplina, para explicar la forma en que los estudiantes atribuyen significado a los símbolos, y para el diseño de secuencias de enseñanza) y también que la presencia - o no - de tales comportamientos en las producciones de los estudiantes permite inferir sobre el grado de desarrollo del sentido de los símbolos.

El ítem 3 también apunta a una negociación de significados, pero requiere, además, poner en juego el marco teórico para brindar explicaciones sobre algo que le es cotidiano al cursillista, a su vez docente: la enseñanza de la matemática, en particular del álgebra. Esperamos que los docentes elaboren una respuesta basada en el plano ideal del deber ser del docente, constructo personal pero que requiere esta instancia de explicitación con otros para construirse, apuntando, así, a la apropiación (PEA, 1993). Además, tal idealización podría materializarse mediante ejemplos de lo que ellos considerarían una práctica que tiene en cuenta este marco teórico. Se insiste en el uso de las referencias en la elaboración grupal para evitar que se convierta en un cúmulo de opiniones personales, pero infundadas, o en un relato de anécdotas que no puedan explicarse ni resolverse mediante constructos de la disciplina.

En el ítem 4 se agrega una fuente más de información, que es la que proviene de la investigación de Fernández (2013). El ítem 4a alude a la apropiación de la caracterización del sentido de los símbolos, profundizando lo preguntado en el ítem 3. 
Después de desarrollar las implicancias del marco teórico para la enseñanza, se espera ahora que los cursillistas reflexionen en torno a los tipos de cambios que demanda el ejercicio de la docencia considerando las conclusiones y recomendaciones didácticas del trabajo de Fernández (2013). Esta propuesta busca que los cursillistas ya no solo interpreten la herramienta y negocien un significado, sino que, además, la usen para proponer cambios en su práctica docente. Se espera que reflexionen sobre el tipo de actividades que proponen y las explicaciones habituales que dan en clase, comparándolas con lo propuesto en el marco teórico de Arcavi y que expliciten las implicancias que una u otra propuesta puede tener sobre el aprendizaje de sus estudiantes.

El ítem 4b fue inspirado en las SCM (MARKOVITS; SMITH, 2008). Exige la selección de una clase concreta, efectivamente dictada por uno de los integrantes, y su reformulación a la luz del constructo del sentido de los símbolos. Por otra parte, se selecciona en particular uno de los comportamientos descriptos por Arcavi (2007) (comportamiento 2: capacidad para manipular y también para leer a través de expresiones simbólicas ${ }^{1}$ ) con el fin de acotar la propuesta para evitar posibles distractores.

Seleccionamos dicho comportamiento porque fue en el que Fernández (2013) detectó mayor aparición de lo que Vinner (2000) llama procedimientos rituales. Esto es, los alumnos resuelven un mismo ejercicio por dos procedimientos distintos. Recién al comprobar que por el segundo procedimiento obtienen la misma solución que por el primero, dan respuesta al ejercicio (FERNÁNDEZ; OCHOVIET, 2015). Además, el comportamiento 2 fue uno de los menos evidenciados en las producciones de los estudiantes de profesorado de matemática. Por estas razones, dado que este curso de posgrado tiene por objetivo que los docentes se especialicen en la enseñanza de la matemática en el nivel superior y, en particular, en la formación de profesores de matemática, nos pareció de relevancia la reflexión sobre este comportamiento.

En síntesis, a los docentes se les proveyó de un marco teórico de referencia que pudiera orientar el diseño de actividades y la gestión del aula, así como también de la situación detectada a nivel del desarrollo del sentido de los símbolos de los estudiantes del último año del profesorado de matemática en un instituto de formación docente de Montevideo. Esto es, conocimiento didáctico del contenido (SHULMAN, 2005).

\footnotetext{
${ }^{1}$ Esto incluye "separarse de los significados y al mismo tiempo adoptar una visión global (gestalt) de las expresiones simbólicas [...] para que las manipulaciones sean relativamente rápidas y eficientes. Por otro lado, la lectura de y "a través" de las expresiones simbólicas con el objeto de captar significados agrega niveles de conexión y razonabilidad a los resultados" (ARCAVI, 2007, p.3).
} 


\section{Análisis de algunas producciones}

Para esta actividad, los cursillistas se agruparon en 13 subgrupos de entre dos y tres integrantes cada uno.

Como nuestro interés reside en dar cuenta de cómo es posible establecer vínculos entre teoría y práctica, nos situamos en el análisis de las producciones de tres equipos de cursillistas referidas a la última parte de la actividad propuesta (parte $4 b$ ).

\subsection{Equipo A (tres integrantes)}

Este equipo de profesores considera para su reformulación una clase de Análisis I, curso del segundo año de la formación inicial de profesores de matemática. La misma aborda conceptos que ya habían sido trabajados previamente como son el de primitiva, función integral y los métodos de integración, en particular el de descomposición en fracciones simples. Para ello proponen la resolución de una ecuación diferencial (Cuadro 1):

\section{Actividad original}

Sea la ecuación $y^{\prime}=\frac{6 x+9}{(3+x) x}$

I) Resuelve dicha ecuación en el intervalo $(0,+\infty)$.

II) Halla la solución $\varphi_{0}$ de la ecuación que pasa por el punto de coordenadas $(1,0)$.

III) Determina la ecuación de la tangente al gráfico de la función $\varphi_{0}$ en el punto de coordenadas $(1,0)$.

Cuadro 1 - Actividad original del equipo A

Fuente: Producción del equipo A de profesores

Los docentes argumentan la elección de esta clase diciendo que: "la actividad que seleccionamos nos parece que se ajusta al comportamiento 2 que menciona Arcavi, ya que para su realización, los alumnos deben analizar, comprender y darle sentido a los símbolos de la actividad planteada" (EQUIPO A). Podemos observar que los docentes introducen una argumentación para la elección de esta clase que va más allá de los contenidos matemáticos a trabajar. Esta se asienta en las metas sugeridas por Arcavi en torno al desarrollo del comportamiento 2. Además, su argumentación es acorde a lo planteado por el grupo en su respuesta a la pregunta $4 \mathrm{a}$ :

Ante la resolución de un problema, es importante estimular a los alumnos a realizar una reflexión previa que les permita decidir cuál es el abordaje más adecuado, usando el álgebra como una herramienta de investigación. En el caso de optar por el uso de los signos [la palabra adecuada es símbolos, esto fue corregido], es fundamental fomentar la inspección de las expresiones simbólicas antes de comenzar a trabajar con ellas, analizando su significado y realizando conjeturas acerca de las posibles soluciones de un problema (EQUIPO A). 
De esta manera, constatamos que los cursillistas fueron capaces de ejemplificar con una propuesta propia lo que describe Arcavi (2007).

Por otra parte, la actividad seleccionada da cuenta de que los profesores se han apropiado de este comportamiento dado que la integral planteada posee una estructura que posibilita el leer a través de los símbolos como bien los profesores lo afirman en el trabajo: "por otro lado, si [los estudiantes] logran "leer a través" de la integral, notarán que $\int \frac{6 x+9}{(3+x) x} d x$ es de la forma $\int \frac{3 g^{\prime}}{g}$, cuya primitiva inmediata es $\ln |g|$, en nuestro caso en el intervalo $R^{+}$(EQUIPO A).

Nos interesa destacar cómo los docentes cambiaron la forma en que estaba planteada la consigna de la actividad, pues de verbos conjugados en forma imperativa se pasó a proponer preguntas que invitan a la investigación de situaciones más que al arribo de "la" respuesta correcta (Cuadro 2):

Actividad reformulada

Sea la ecuación (e): $y^{\prime}=\frac{6 x+9}{(3+x) x}$

1.

i) ¿Por qué la ecuación (e) tiene solución en el intervalo $(0,+\infty)$ ? Explicita la propiedad o propiedades usadas.

ii) ¿La solución es única? Justifica tu respuesta y si es afirmativa, responde: ¿qué dato agregarías al enunciado para obtener la unicidad de la solución?

iii) De los siguientes campos de direcciones, ¿cuál puede corresponder al de la ecuación (e)?

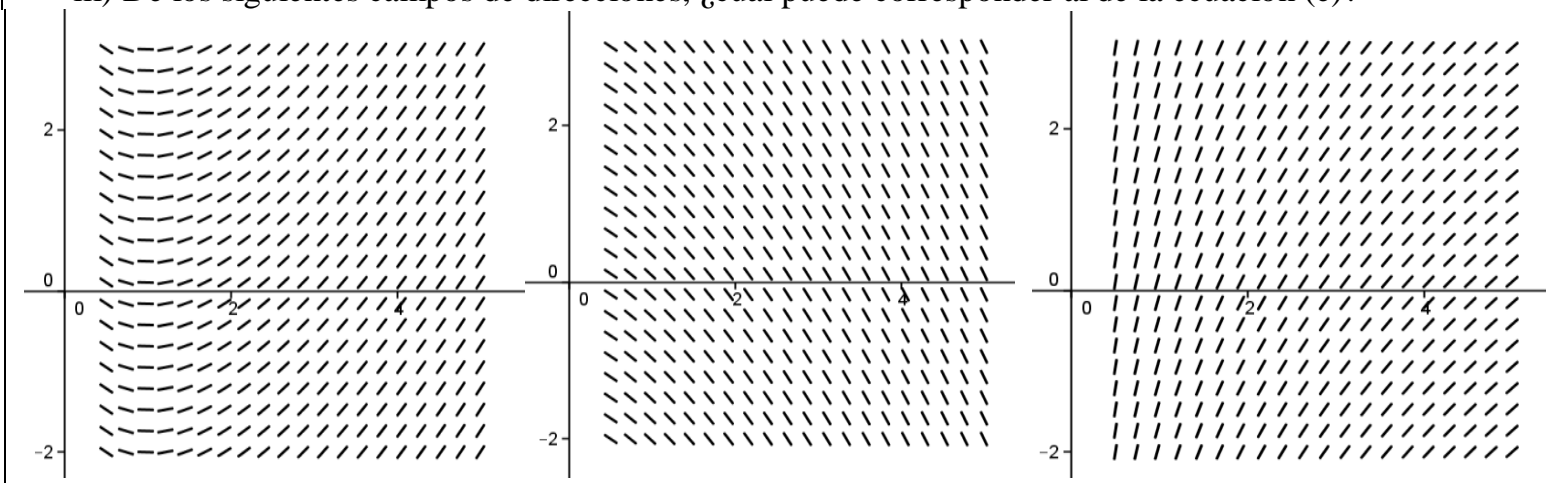

2. Resuelve dicha ecuación en el intervalo $(0,+\infty)$, empleando dos procedimientos distintos. ¿Cuál de ellos consideras más conveniente? Justifica.

3. Halla la solución $\varphi_{0}$ de la ecuación que pasa por el punto de coordenadas $(1,0)$.

4. Determina la ecuación de la tangente al gráfico de la función $\varphi_{0}$ en el punto de coordenadas $(1,0)$.

Cuadro 2 - Actividad reformulada del equipo A

Fuente: Producción del equipo A de profesores

Las preguntas 1 i), 1 ii) y 1 iii) agregadas previamente a la actividad original (partes 2, 3 y 4) reflejan una inquietud de los docentes por proponer actividades que promuevan el comportamiento en cuestión, dado que en lugar de pedir directamente que se resuelva la ecuación, se solicita que se reflexione sobre la existencia y cantidad de soluciones. Incluso 
aclaran, en la fundamentación, la posibilidad de una intervención específica en ese sentido en caso de ser necesaria: "puede ocurrir que directamente decidan manipular la expresión y resuelvan la ecuación, en dicho caso el docente deberá intervenir para interrumpir ese proceso mecánico y reflexionar que no deben abalanzarse sobre el problema sino analizarlo" (EQUIPO A).

Nuevamente estas reflexiones por parte del grupo reflejan la capacidad de poner en práctica concreta de aula algunos de los aportes de Arcavi.

Es interesante la inclusión de la consigna 1 iii) respecto al posible campo de direcciones correspondiente a la ecuación. En primer lugar, porque no es habitual un abordaje gráfico para reconocer posibles representaciones de la solución. Las prácticas tradicionales se enfocan en resoluciones analíticas y precisas. Por otro lado, se aprecia nuevamente la intencionalidad de lograr que los estudiantes lean a través de los símbolos, vinculándolos con sus representaciones gráficas. Para resolverlo es necesario que el estudiante reconozca que el miembro derecho de la expresión representa la función derivada de las funciones soluciones y que por tanto analizar su signo puede dar respuesta acerca de cómo debe ser el campo de direcciones de la ecuación.

Nuevamente se evidencia el interés por priorizar la inspección del significado de los símbolos sobre la manipulación algebraica rutinaria, como ellos mismos expresan: "en las tres partes de la actividad, un análisis previo de la situación permitiría optar por un camino más corto, evitando el uso de herramientas más complejas en forma innecesaria" (EQUIPO A). Por otra parte, la intención de los cursillistas de que los estudiantes recurran a las representaciones gráficas, en un caso oportuno, fomenta la transición flexible entre diferentes representaciones. Lo que, según Arcavi (1994) enriquece la comprensión de cada representación y de la misma manera el sentido de los símbolos.

Finalmente, los profesores dan cuenta del rediseño de las consignas, explicitando una intención didáctica que pretende dar lugar a la aparición intencional de distintos procedimientos en lugar de dejarlo librado a la aparición de estrategias espontáneas por parte de los estudiantes. Expresan que:

En la parte 2 se agrega la consigna de pensar en dos procedimientos distintos. Probablemente algunos estudiantes usarían procedimientos diferentes para descomponer en fracciones simples, otros emplearían el segundo procedimiento y la descomposición en fracciones simples y probablemente alguno encuentre alguna opción que no mencionamos. Se podría pensar que no hay diferencia con la propuesta original, en la que se pueden discutir las distintas formas de resolución en la puesta en común. Pero al pedir que se resuelva la ecuación por dos procedimientos distintos, el alumno percibe que hay más de una forma de resolver la misma ecuación, y se ve obligado a analizar la situación, buscando diferentes posibilidades (EQUIPO A). 
En suma, muchos aspectos de la elaboración grupal permiten sugerir que los profesores cursillistas no solo negociaron significados relativos a la propuesta de Arcavi (1994, 2007) y Fernández (2013) sino que, además, se apropiaron de ellos, reelaborando una propuesta concreta de aula en función de tales significados.

\subsection{Equipo B (tres integrantes)}

Estos profesores consideran para reformular, una clase del curso Geometría y Álgebra Lineal, del segundo año de la formación inicial de profesores de matemática, en la que se pretende abordar la condición necesaria y suficiente para que un conjunto sea un subespacio de un espacio vectorial. En clases anteriores se había trabajado con los conceptos de espacio y subespacio vectorial y analizaron junto a los alumnos cuáles son los subespacios de $\mathrm{R}^{2}$ y de $\mathrm{R}^{3}$. A partir de este análisis, en la clase propuesta originalmente se planteó la condición, enunciando el respectivo teorema y demostrándolo junto a los alumnos. A continuación de la demostración del teorema se propuso el siguiente ejercicio (Cuadro 3):

Actividad original

Averigua si los siguientes conjuntos son subespacios vectoriales del espacio vectorial V indicado en cada caso.

1) $S_{1}=\left\{(x, y) \in R^{2}: x=y\right\}$ y $V=R^{2}$

2) $S_{2}=\left\{(x, y) \in R^{2}: x=y^{2}+y\right\}$ y $V=R^{2}$

3) $S_{3}=\left\{(x, y) \in R^{2}: x \in\{0,1,2,3,4\}\right\}$ y $V=R^{2}$

4) $S_{4}=\left\{(x, y, z) \in R^{3}:(x-1)^{2}=(x+y)^{2}\right\}$ y $V=R^{3}$

5) $S_{5}=\left\{P \in V: a_{0}=4\right\}$ y $V$ el espacio vectorial de los polinomios de variable real, con coeficientes reales de grado 2 , de la forma $P(x)=a_{2} x^{2}+a_{1} x+a_{0}$.

\section{Cuadro 3 - Actividad original del equipo B \\ Fuente: Producción del equipo B de profesores}

Los propios docentes reflexionan sobre esta forma de trabajo señalando que: "al plantear estos ejercicios inmediatamente después del teorema, la propuesta provocó que los estudiantes los resolvieran utilizando procedimientos algebraicos de forma mecanizada, sin inspeccionar cada situación en su contexto" (EQUIPO B). Como podemos observar, los aportes teóricos de Arcavi son utilizados para analizar didácticamente su propuesta, les permite posicionarse críticamente sobre la secuenciación elegida y, además, se apropian del vocabulario específico del marco y lo utilizan adecuadamente.

En la clase reformulada, si bien el objetivo sigue siendo abordar la condición necesaria y suficiente para que un conjunto sea un subespacio de un espacio vectorial, sugieren un 
cambio en la secuenciación de contenidos. Proponen comenzar la clase con el planteo del ejercicio que en la clase original se había propuesto a continuación de la demostración del teorema.

Esperan que algunas de las situaciones sean analizadas desde una perspectiva geométrica de los conjuntos dados aunque reconocen que, tanto procedimientos sui generis como la evocación de la propia definición podrían ser utilizados por parte de los alumnos. En otras de las situaciones planteadas, la interpretación geométrica resulta más compleja por lo que piensan que los estudiantes recurrirán a la definición. Señalan que:

[...] el docente intervendrá en esta instancia haciendo énfasis en la importancia de la lectura de los símbolos para una más eficiente selección del procedimiento a realizar, por ejemplo en el ejercicio 4 reconocer que la interpretación gráfica de dicho conjunto no es tan evidente, y sí lo es el reconocer que el elemento nulo no pertenece al conjunto (EQUIPO B).

Luego de este trabajo práctico, los docentes creen que: "a partir de lo realizado en estos ejercicios se verá qué aspectos de la definición son importantes verificar para identificar un subespacio vectorial, a partir de lo cual se enunciará el siguiente teorema” (EQUIPO B), refiriéndose a la condición necesaria y suficiente, ya mencionada anteriormente.

La reformulación de una clase que ya fue dictada se encuentra bien fundamentada en el marco teórico de referencia y pone en juego el comportamiento 2 de Arcavi en tanto los docentes sugieren poner en tensión interpretaciones geométricas y manipulaciones algebraicas para contribuir a delimitar las situaciones en las que esa manipulación es ineludible. Para lograr esto, la inspección previa de los símbolos es requerida.

Lo que resulta más interesante de la propuesta y que da cuenta de la apropiación del marco teórico como elemento que da dirección a las decisiones didácticas, lo constituye la situación final de reinversión, propuesta después del enunciado y demostración de la condición necesaria y suficiente para que un conjunto sea un subespacio de un espacio vectorial (Cuadro 4):

Actividad reformulada

Determina si los siguientes conjuntos son subespacios vectoriales del espacio vectorial considerado en cada caso. Discute con tu compañero si hay más de un procedimiento para averiguarlo y explica cuál les parece más conveniente en cada caso.

A) $S_{1}=\left\{(x, y) \in R^{2} ; y=m x+n\right\}$ con $m, n \in R$

B) $S_{2}=\{f \in A ; f(1) \cdot f(0)<0\}$ siendo $A=\{f: R \rightarrow R / f$ función lineal $\}$

C) $S_{3}=\left\{(x, y, z) \in R^{3} ; z=0\right\}$

D) $S_{4}=\left\{P \in P_{3} ; a_{0}+a_{1}+a_{2}+a_{3}=0\right\}$ con $P(x)=a_{3} x^{3}+a_{2} x^{2}+a_{1} x+a_{0}$

Cuadro 4 - Actividad reformulada del equipo $\mathrm{B}$

Fuente: Producción del equipo B de profesores 
Si bien los estudiantes ya cuentan con la información que provee la condición necesaria y suficiente, se sigue pensando no en una aplicación automática de esta condición sino que se jerarquizan en simultáneo la diversidad de estrategias y habilidades que podrían ser puestas en juego, como los propios docentes explicitan:

Aquí se pretenderá analizar junto con los estudiantes que en $S_{1}$ se ve la necesidad de interpretar geométricamente, discutiendo el valor de $m$ y $n$; mientras que en $S_{2}$ si se realiza una correcta lectura de los símbolos se verá que la función nula no pertenece al conjunto, por ende no es un subespacio; en $S_{3}$ se podrá analizar geométricamente o aplicar el teorema que vimos para determinar si es o no un subespacio, pero la interpretación geométrica quizás sea más 'directa'; y por último, en $S_{4}$ se discutirá sobre la necesidad de utilizar el teorema ya que no es fácil de visualizar una interpretación gráfica del conjunto y si el elemento nulo pertenece al mismo (EQUIPO B).

\subsection{Equipo C (dos integrantes)}

La clase a reformular corresponde a la asignatura Cálculo de la carrera de Ingeniería Audiovisual de una universidad privada. La clase tuvo por objetivo que el estudiante fuera capaz de determinar el dominio de una función compuesta. Se planteó la siguiente actividad (Cuadro 5):

\section{Actividad original}

En cada caso, halla $(f \circ g)$ y $(g \circ f)$ y sus dominios respectivos:

$$
\begin{array}{rlrl}
\text { a) } f: f(x)=4 & \text { c) } f: f(x)=\sqrt{x} \\
g: g(x)=-2 x^{2}-6 x & g: g(x)=1-x^{2} \\
\text { b) } f: f(x)=2 x+5 & \text { d) } f: f(x)=\left\{\begin{array}{l}
2 x-5 \text { si } x<2 \\
x^{2}-4 x+3 \text { si } x \geq 2
\end{array}\right. \\
g: g(x)=x^{2}+3 & g: g(x)=2 x+3
\end{array}
$$

Cuadro 5 - Actividad original del equipo C Fuente: Producción del equipo $\mathrm{C}$ de profesores

Las docentes relatan que la clase comenzó con un repaso de la definición de función compuesta. Los alumnos trabajaron en el problema y el docente fue atendiendo dudas. Luego de transcurridos veinte minutos se comenzó con la puesta en común en la que se discutieron las estrategias empleadas y los resultados a los que se arribaron.

En la clase reformulada, se propone la siguiente actividad (Cuadro 6): 


\section{Actividad reformulada}

Considere las funciones reales $f:[-4,+\infty) \rightarrow R, f(x)=\sqrt{x+4}$ y $g:[-2,+\infty) \rightarrow R, g(x)$ es un polinomio de segundo grado. Se adjunta representación gráfica de $g$.

Hallar, si es posible, el dominio de la función $f \circ g$.

Cuadro 6 - Actividad reformulada del equipo C

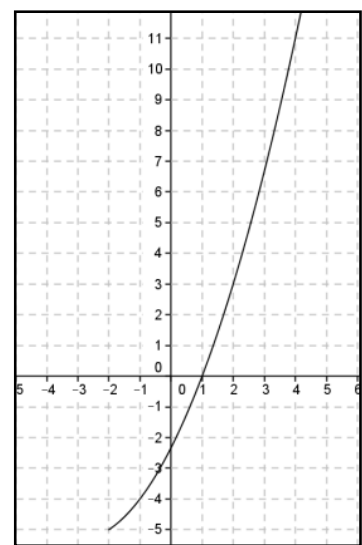

Fuente: Producción del equipo $\mathrm{C}$ de profesores

En cuanto a lo metodológico, vuelven a señalar que primero los estudiantes trabajarán en el problema y el profesor los asistirá disipando dudas para luego realizar una puesta en común.

Las docentes dan cuenta de que ahora consideraron poner en juego el comportamiento 2 de Arcavi, cuando explicitan cómo se realizará la puesta en común, aspecto que no fue jerarquizado en la clase original. En el análisis a priori conjeturan que seguramente los alumnos abordarían el problema averiguando "la expresión analítica de $g$ con una secuencia de procedimientos mecánicos a partir de datos extraídos de la representación gráfica" (EQUIPO C). Pero agregan que modelaron la función $g$ de modo que no animara a los estudiantes a seguir ese camino. Obsérvese que a diferencia de la actividad original, en este caso la expresión analítica de $g$, no está dada. Las docentes plantearon que esto fue hecho con la intención de que surgiera en los estudiantes la idea de que no es necesaria una manipulación mecánica de las expresiones analíticas, sino que es suficiente con el análisis de la definición del dominio de la función compuesta. Agregan que si se aplica la definición de dominio de una función compuesta, se llega a que $D(f \circ g)=\{x \in D(g) / g(x) \in D(f)\}$, entonces $D(f \circ g)=\{x \in[-2,+\infty) / g(x) \in[-4,+\infty)\}$ y que la inspección de esta última expresión articulada con la representación gráfica dada, permitirá por un lado dar respuesta a la situación planteada y por otro, poner en evidencia que la obtención de la expresión analítica de $g$ no es necesaria. Las docentes agregan que:

Esta actividad brinda una excelente oportunidad de interrumpir el uso automatizado de procedimientos para dejar en evidencia que la inspección a priori, durante y después de los símbolos permite analizar el sentido de la consigna para alcanzar su resolución. Este análisis es esencial debido a que de no hacerlo el estudiante caería en una sucesión de cálculos más complejos de los necesarios para resolver la situación planteada (EQUIPO C).

Entendemos que en este equipo hubo una adecuada apropiación del marco teórico propuesto y que ello queda evidenciado en la reformulación de la tarea original al omitir dar 
la expresión analítica de una de las funciones y sustituirla por la expresión gráfica. Esto es lo que habilita a los alumnos a realizar la inspección de la situación para dar respuesta y bloquea la manipulación simbólica sin sentido.

\section{Reflexiones finales}

La actividad diseñada con el objetivo de que los profesores comprendieran y se apropiaran del marco teórico de Arcavi alcanzó, en buena medida, sus objetivos en tanto a través de la reformulación de una clase que ya había sido dada, los docentes pudieron mostrar diseños de actividades con el fin de poder capitalizar la lectura de los símbolos.

Tal fue el caso de una clase que se centró en la resolución de una ecuación diferencial con una estructura coherente con ese fin, la cual los docentes reelaboraron agregando preguntas que invitaban a la inspección de los símbolos. Por su parte, el grupo que trabajó con la condición necesaria y suficiente para que un conjunto sea un subespacio de un espacio vectorial, optó por reorganizar la secuenciación de las actividades de la clase con el propósito de que a través de la lectura de los símbolos y de la mediación con representaciones gráficas pudieran deducirse condiciones para la elaboración de una conjetura, que luego sería sometida a prueba.

En el caso de las docentes que trabajaron con un problema que requería hallar el dominio de una función compuesta, la intervención en la propuesta consistió en cambiar el registro de representación de una de las funciones dadas como dato inicial: de algebraico a gráfico. Esa modificación invita a los estudiantes a pensar alternativas de resolución diferentes a las manejadas en tareas tradicionales, conduciéndolos a una inspección de los símbolos para evitar cálculos innecesarios (como podría ser el hallar la expresión analítica de la función a partir de su representación gráfica).

En todos los casos detectamos que la actividad propuesta a los docentes permitió imprimir a la reformulación de las clases una intencionalidad didáctica sustentada en un marco teórico, así como la apropiación del vocabulario específico del marco. Lo que proponemos como un nuevo tipo de SMC (MARKOVITS; SMITHS, 2008), que consistió en recuperar una situación real de clase y reelaborarla, resultó muy efectivo a los efectos de generar aprendizajes desde la perspectiva sociocultural propuesta (PEA, 1993). Consideramos que, a partir de diseños como el que utilizamos, es posible contribuir a la comprensión de los resultados de investigación así como a lograr vínculos entre investigación y práctica docente. 


\section{Referencias}

ARCAVI, A. Symbol sense: informal sense-making in formal mathematics. For the learning of mathematics, Fredericton, v. 14, n. 3, p. 24-35, nov. 1994.

ARCAVI, A. El desarrollo y el uso del sentido de los símbolos. Conferencia realizada como profesor visitante, Criced, Tsukuba University- Japan. 2007. Diponible en: $\lfloor$ http://srvcnpbs.xtec.cat/c reamat/joomla/images/stories/documents/visualizacio/arcavi_desarrollo.pdf $>$ Acceso en: 22 may. 2015.

BOERO, P.; SZENDREI, J. R. Research and results in mathematics education: some contradictory aspects. In: SIERPINSKA, A.; KILPATRICK, J. (Ed.), Mathematics education as a research domain: a search for identity. The Netherlands: Kluwer academic publishers, 1998. p. 197-212.

BORKO, H. Professional development and teacher learning: mapping the terrain. Educational researcher, New York, v. 33, n. 8, p. 3-15, nov. 2004.

BRITT, M. S.; IRWIN, K. C.; RITCHIE, G. Professional conversations and professional growth. Journal of Mathematics Teacher Education, Reston, v. 4, n. 1, p. 29-53, 2001.

CANTORAL, R. ¿Publicar o perecer, o publicar y perecer? Revista Latinoamericana de Matemática Educativa, México, v. 10, n. 3, p. 311-313, 2007.

CASTRO, A.; LI, W. Developing Mathematical Knowledge for Teaching Teachers: a Model for the Professional Development of Teacher Educators. Issues in Teacher Education, Orange, v. 23, n. 1, p. 113-132 2014.

CZARNOCHA, B.; PRABHU, V. Teaching-research and design experiment - two methodologies of integrating research and classroom practice. In: EPISTEME-1, INTERNATIONAL CONFERENCE TO REVIEW RESEARCH ON SCIENCE, TECHNOLOGY AND MATHEMATICS EDUCATION, 1. 2004, Goa. Proceedings... Goa, 2004, p. 78-80. Available at:

<http://www.hbcse.tifr.res.in/episteme/episteme-1/themes/OP Czarnocha PrabhuModified.pdf.> Accessed on: 14 aug. 2014.

DESGAGNÉ, S.; BEDNARZ, N.; LEBUIS, P.; POIRIER, L.; COUTURE, C. L’approche collaborative de recherche en éducation: un rapport nouveau à établir entre recherche et formation. Revue des sciences de l'éducation, Montreal, v. 27, n. 1, p. 33-64 2001.

EVEN, R. Integrating academic and practical knowledge in a teacher leaders' development program. Educational studies in mathematics, The Netherlands, v. 38, n. 1, p. 235-252, mar. 1999.

FERNÁNDEZ, J. El desarrollo del sentido de los símbolos en la formación inicial de profesores de matemática. Reflexiones en torno a la enseñanza del álgebra. 2013, 402f., Tesis (Maestría en Enseñanza de las Ciencias Naturales y Exactas), Universidad Nacional del Comahue, Neuquén, 2013.

FERNÁNDEZ, J.; OCHOVIET, C. Procedimientos rituales en la resolución de ejercicios en contexto algebraico en estudiantes de profesorado de matemática. Bolema, Rio Claro, v. 29, n. 52, p. 704-728, ago. 2015.

GRESALFI, M. S.; COBB, P. Negotiating identities for mathematics teaching in the context of professional development. Journal for Research in Mathematics Education, Reston, v. 42, n. 3, p. 270-304, 2011.

GUSKEY, T. R. Professional development and teacher change. Teachers and Teaching: theory and practice, v. 8, n. 3, p. 381-391, 2002. 
MARKOVITS, Z.; SMITH, M. Cases as tools in mathematics teacher education. In: WOOD, T.; TIROSH, D. (Ed.), International Handbook of Mathematics Teacher Education, 2: Tools and Processes in Mathematics Teacher Education. Rotterdam, The Netherlands: Sense Publishers 2008, p. $39-64$.

MONTECINOS, C. Desarrollo profesional docente y aprendizaje colectivo. Psicoperspectivas. Individuo y Sociedad, Valparaíso, v. 2, n. 1, p. 105-128, 2003.

MOSCHKOVICH, J.; BRENNER, M. E. Using a naturalistic lens on mathematics and science cognition and learning. In: KELLY, A.; LESH, R. (Ed.), Research design in mathematics and science education. Mahwah: Erlbaum, 2000. p. 457-486.

NIPPER, K.; SZTAJN, P. Expanding the instructional triangle: conceptualizing mathematics teacher development. Journal of Mathematics Teacher Education, Reston, v. 11, n. 4, p. 333-341, ago. 2008.

OCHOVIET, C. Una lectura del estado de la investigación en educación matemática y un "por aquí cómo andamos". Conversación, Montevideo, v. 19, p. 45-50, 2007.

OCHOVIET, C.; OKTAÇ, A. Comprender los resultados de investigación: labor docente del investigador en la enseñanza de la matemática educativa. In: BUENDÍA, G. (Coord.), Reflexión e investigación en matemática educativa. México: Lectorum, 2011. p. 53-80.

OCHOVIET, C.; VIEITEZ, J. Plan de estudios del posgrado diploma en matemática (mención aplicaciones o mención enseñanza). Consejo de formación en educación (ANEP) y Universidad de la república (UdelaR). Uruguay. Documento oficial no publicado. 2012.

PEA, R. Learning scientific concepts through material and social activities: conversational analysis meets conceptual change. Educational psychologist, Philadelphia, v. 28, n. 3, p. 265-277, 1993.

RAYMOND, A.; LEINENBACH, M. Collaborative action research on the learning and teaching of algebra: a story of one mathematics teacher's development. Educational studies in mathematics, The Netherlands, v. 41, n. 3, p. 283-307, mar. 2000.

SHULMAN, L. Conocimiento y enseñanza: fundamentos de la nueva reforma. Revista de currículum y formación del profesorado, Granada, v. 9, n. 2, p. 1-31, 2005.

TSAMIR, P. Using theories as tools in mathematics teacher education. In: TIROSH, D.; WOOD, T. (Ed.) The international handbook of mathematics teacher education volume 2: tools and processes in mathematics teacher education. Rotterdam: Sense Publishers, 2008. p. 211-234.

VINNER, S. Mathematics education - procedures, rituals and man's search for meaning. Conferencia dictada en el ICME 9, Universidad Ben Gurion de El Negev, Japón. 2000. Available at: 〈http://www.fisme.science.uu.nl/nwd/nwd2003/handouts/vinner. pdf > Accessed on: 17 aug. 2014.

WASSERMAN, S. El estudio de casos como método de enseñanza. Buenos aires: Amorrortu, 1999.

Submetido em Abril de 2015. Aprovado em Junho de 2015. 


\section{Anexo 1 - Diseño de la actividad propuesta en el curso}

1) Realicen la lectura del documento: Arcavi, A. (2007) El desarrollo y el uso del sentido de los símbolos. Conferencia realizada como profesor visitante, criced, tsukuba university- japan. En http://ebookbrowse.com/arcavi05-el-desarrollo-y-el-uso-del-sentido-de-los-simbolos-doc-

d37871752 (recuperado el 28/04/2014)

Si es posible, lean también:

Arcavi, A. (1994). Symbol sense: informal sense-making in formal mathematics. For the learning of mathematics. 14(3), pp. 24-35. En

http://www.fisme.science.uu.nl/fisme/nl/projecten/minisymposiumalgebraict/arcavi1994flm.pdf

(recuperado el 28/04/2014)

2) Expliquen en un máximo de 200 palabras (Word contiene una herramienta para contar palabras), en qué consiste, según Arcavi, "el sentido de los símbolos".

3) En su consideración, ¿qué implicancias tienen en la enseñanza los planteos que realiza Arcavi en torno al desarrollo del sentido de los símbolos? Se espera una elaboración grupal pero sostenida en los documentos de referencia (250 palabras máximo).

4) Les proponemos ahora realizar la lectura de algunas secciones de la tesis de maestría: Fernández, J. (2013). El desarrollo del sentido de los símbolos en la formación inicial de profesores de matemática. Reflexiones en torno a la enseñanza del álgebra. Tesis de maestría no publicada, Universidad Nacional del Comahue. Neuquén - Argentina: Capítulo 3, Capítulo 5, las "respuestas de Clara" en las páginas 134 a 150 y de la página 352 a la 377.

Los sujetos entrevistados en esta investigación fueron todos los estudiantes de cuarto año de la especialidad matemática, de un instituto de formación docente, que tenían grupo de práctica a cargo.

a) Este estudio tiene implicancias acerca del trabajo que realizamos como docentes en la formación de profesores de matemática. Planteen una reflexión grupal acerca del tipo de cambios que demanda el ejercicio de la docencia en ese nivel y en esa especialidad, a la luz de las conclusiones y las recomendaciones didácticas del trabajo. (Máximo una carilla A4)

b) Seleccionen una clase de 45 minutos que alguno de los integrantes haya dado alguna vez (en magisterio o en el profesorado de matemática), en la que ahora se den cuenta que podría estar implicado el comportamiento 2 de Arcavi, según fue considerado en la tesis analizada. ¿Cómo modificarían esa clase (tanto las actividades, su secuenciación, el discurso del docente y sus intervenciones) para que la misma diera lugar a trabajar con el "sentido de los símbolos" particularmente lo que refiere al comportamiento 2? En una carilla relaten brevemente la clase tal como fue dada y en tres carillas máximo, cómo la reformularían.

Tengan en cuenta que los comportamientos descriptos por Arcavi son constructos que podemos utilizar los docentes para analizar y comprender lo que los estudiantes aprenden pero de ninguna manera constituyen objetivos de enseñanza. Vale decir, no tendría sentido decir en una clase: "hoy vamos a estudiar el sentido de los símbolos" o "en la clase de hoy vamos a desarrollar la capacidad para "manipular" y también para "leer a través" de expresiones simbólicas". Lo que estamos estudiando son conceptos de la didáctica de la matemática que nos sirven, desde nuestro rol como docentes, para comprender y tomar decisiones acerca de la enseñanza pero de ninguna manera constituyen cuestiones para enseñar a nuestros alumnos, al menos en nuestras clases de matemática. 